American Journal of Internal Medicine
2015;3(3): $141-145$
Published online May 4, 2015 (http://www.sciencepublishinggroup.com/j/ajim)
doi: $10.11648 /$ jajim.20150303.18
ISSN: 2330-4316 (Print); ISSN: 2330-4324 (Online)

\title{
An Unusual Source of Systemic Thromboembolism
}

\author{
Olusegun Sheyin ${ }^{1}$, Bredy Pierre-Louis ${ }^{2}$ \\ ${ }^{1}$ Department of Medicine, Harlem Hospital Center, Columbia University, College of Physicians and Surgeons, New York, USA \\ ${ }^{2}$ Division of Cardiology, Department of Medicine, Harlem Hospital Center, Columbia University, College of Physicians and Surgeons, New \\ York, USA
}

Email address:

oas2120@cumc.columbia.edu (O. Sheyin)

\section{To cite this article:}

Olusegun Sheyin, Bredy Pierre-Louis. An Unusual Source of Systemic Thromboembolism. American Journal of Internal Medicine. Vol. 3, No. 3, 2015, pp. 141-145. doi: 10.11648/j.ajim.20150303.18

\begin{abstract}
Pulmonary vein thrombosis (PVT) is a rare but potentially life threatening condition. This is a case of pulmonary vein thrombosis presenting with systemic thromboembolism despite treatment with anticoagulation. A 73-year-old woman, with past medical history of hypertension, peripheral arterial disease (PAD), and treated endometrial and breast cancer, presented with complaint of pain in her right foot of one-day-duration. She had a history of PVT diagnosed on chest CT angiogram one month prior to presentation, which was done to exclude pulmonary embolism when she presented with progressively worsening dyspnea. Physical examination was notable for an elderly woman in painful distress, with a cold, pulseless right foot with bluish discoloration of the skin of the forefoot and a clear line of demarcation from the proximal foot. The angiogram revealed occlusion of the right popliteal artery just above the knee with no appreciable blood flow within the distal vessels. Contrast CT of the abdomen and pelvis revealed multiple hypo-enhancing ill-defined hepatic masses, bilateral adrenal masses and mediastinal adenopathy. Echocardiogram revealed a large left ventricular thrombus and hyper-dynamic left ventricular systolic function. She was admitted for acute right foot ischemia and underwent popliteal exploration, open thrombectomy and embolectomy with restoration of pedal flow. However, she had repeat right popliteal exploration two days later due to further ischemic changes in the foot. Post-operatively, she developed multi-organ dysfunction including respiratory failure requiring endotracheal intubation and mechanical ventilation. She was terminally weaned from mechanical ventilation on request by her family and she died on the sixth day of admission. This case describes the occurrence of systemic embolization despite anticoagulation for pulmonary vein thrombosis. The large thrombus found in the left ventricle most likely represents a thrombus in transit from the superior left pulmonary vein en-route systemic embolization with consequent multiorgan dysfunction.
\end{abstract}

Keywords: Pulmonary Vein Thrombosis, Peripheral Arterial Disease, Acute Ischemia, Thrombectomy, Embolectomy

\section{Introduction}

Pulmonary vein thrombosis (PVT) is a rare but potentially life threatening condition ${ }^{[1]}$. Few cases have been reported describing the occurrence of PVT in patients undergoing surgeries such as lung transplantation or lobectomy; and in patients with sclerosing mediastinitis, primary or secondary tumors of the lung and atrial myxoma ${ }^{[2-7]}$. PVT can present either acutely as pulmonary infarction with cough, dyspnea and pleuritic chest pain or in a more insidious manner as progressive or recurrent pulmonary edema and pulmonary fibrosis ${ }^{[1,2]}$. Prompt diagnosis of PVT is desirable to prevent or limit systemic thromboembolization ${ }^{[7]}$. However, because of its non-specific signs and symptoms, PVT is difficult to diagnose clinically. This report is a case of pulmonary vein thrombosis presenting with systemic thromboembolism despite treatment with anticoagulation.

\section{Case Report}

A 73-year-old woman, ex-smoker with 40 pack-years of smoking presented with complaint of pain in her right foot of one-day-duration. She has past medical history of hypertension, peripheral arterial disease (PAD), endometrial cancer diagnosed fifteen years ago, treated with hysterectomy and radiotherapy; and invasive ductal carcinoma of the left breast diagnosed ten years ago and treated with lumpectomy, radiation and chemotherapy. The patient had earlier presented one month ago with progressively worsening dyspnea and abdominal pain, two weeks after a one-month hospitalization 
at another hospital for dyspnea, where she had thoracocentesis and chest tube placement for pleural effusion, in addition to liver biopsy and left femoral-femoral bypass. She was found to have acute pancreatitis and pulmonary vein thrombosis on chest CT angiogram, which was done to exclude pulmonary embolism. She was started on subcutaneous enoxaparin $80 \mathrm{mg}$ every twelve hours and was managed conservatively for acute pancreatitis. The patient was discharged on the same dose of enoxaparin, which she reported good compliance with. She was otherwise in her usual state of health until she developed the right foot pain at rest, with no swelling or prior history of trauma or gout. She became concerned when the pain was not remitting with acetaminophen and she noted that the foot was becoming cold and dusky in color. Hence, she presented to the emergency room.

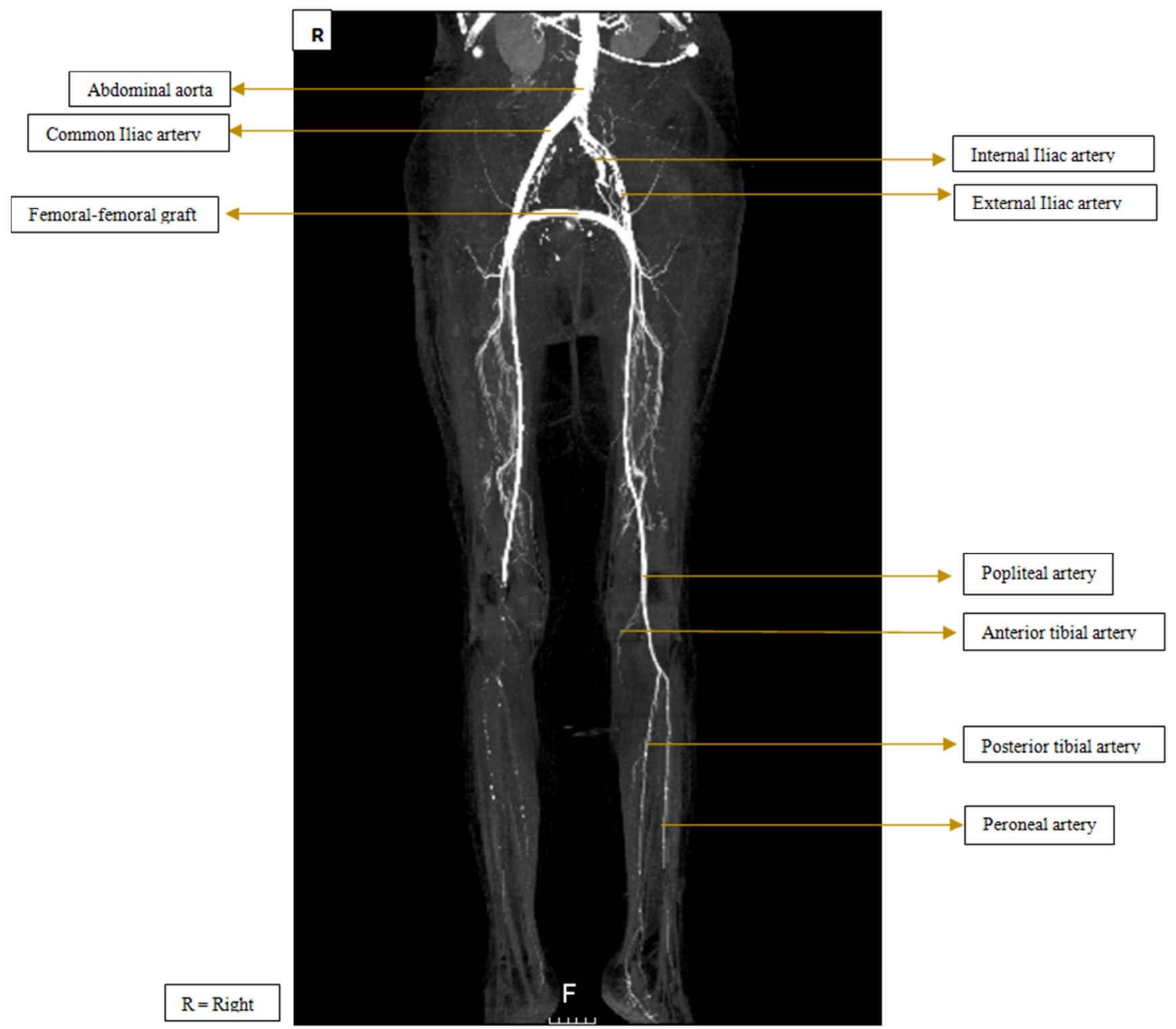

Figure 1. CT angiogram of the arterial system of the lower extremities revealing occlusion of the right popliteal artery just above the knee.

Vital signs on presentation were blood pressure of $121 / 99 \mathrm{mmHg}$, pulse $121 /$ minute, respiratory rate $24 /$ minute, and temperature $97.5^{\circ} \mathrm{F}$ with oxygen saturation $100 \%$. Physical examination was notable for an elderly woman in painful distress, with dry mucous membranes and mild pallor, without icterus, central cyanosis or evidence of respiratory distress. She had normal first and second heart sounds and decreased breath sounds in the left lower lung zone. The right foot was cold, pulseless and it had a bluish discoloration of the skin extending distally from the metatarso-phalangeal joints, with a clear line of demarcation from the proximal foot, which was warm and had normal skin color. Complete blood count revealed white blood cell count of 11,200 per microliter, hemoglobin of $9.2 \mathrm{mg} / \mathrm{dL}$ and a platelet count of 119,000 per microliter. Renal function was normal on admission, with serum creatinine of $1.0 \mathrm{mg} / \mathrm{dL}$.

Bilateral venous doppler ultrasound of the lower extremities was negative for deep vein thrombosis down to the level of the right and left popliteal veins. She had a CT angiogram which revealed occlusion of the right popliteal 
artery just above the knee with no appreciable flow within the right anterior tibial, peroneal and posterior tibial arteries. The CT angiogram also revealed patent femoral-femoral bypass and occlusion of the right internal iliac artery, left common iliac artery, left proximal internal and left external iliac arteries (Figure 1). Her chest X-ray revealed multiple pleural-based masses and nodules in the left hemithorax and a moderate-sized left pleural effusion (Figure 2). Chest CT with contrast revealed no filling defects within the main, lobar, or segmental pulmonary arteries to suggest a pulmonary arterial embolism. However, there was a filling defect within the superior left pulmonary vein consistent with a venous thrombus. Other findings on the chest CT include bulky mediastinal and left hilar adenopathy, multiple pleural nodules, and left thyroid mass, without pulmonary consolidation, pleural effusion, or pneumothorax (Figure 3). Contrast CT of the abdomen and pelvis revealed multiple hypoenhancing ill-defined hepatic masses, bilateral adrenal masses, mediastinal adenopathy, left anterior abdominal wall nodule and mild fusiform dilatation of infra-renal aorta up to $2.6 \mathrm{~cm}$ with thrombosis of the left common iliac, proximal internal iliac, and external iliac arteries (Figure 4). Echocardiogram revealed a large left ventricular mass consistent with thrombus and a left ventricular ejection fraction of $80 \%$ (Figure 5).

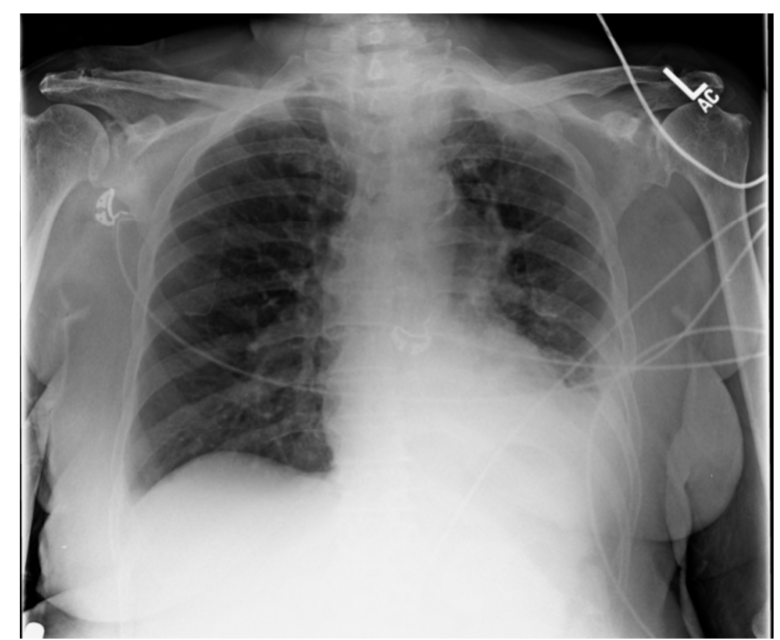

Figure 2. Chest X-ray showing multiple pleural-based masses and nodules in the left hemithorax and a moderate-sized left pleural effusion.

She was admitted for acute right foot ischemia and underwent popliteal exploration, open thrombectomy and embolectomy with restoration of pedal flow. However, she had further ischemic changes to her right foot; hence she was taken back to surgery two days later for right popliteal exploration, intraoperative angiography, and selective injection of tissue plasminogen activator into the right anterior and posterior tibial arteries.

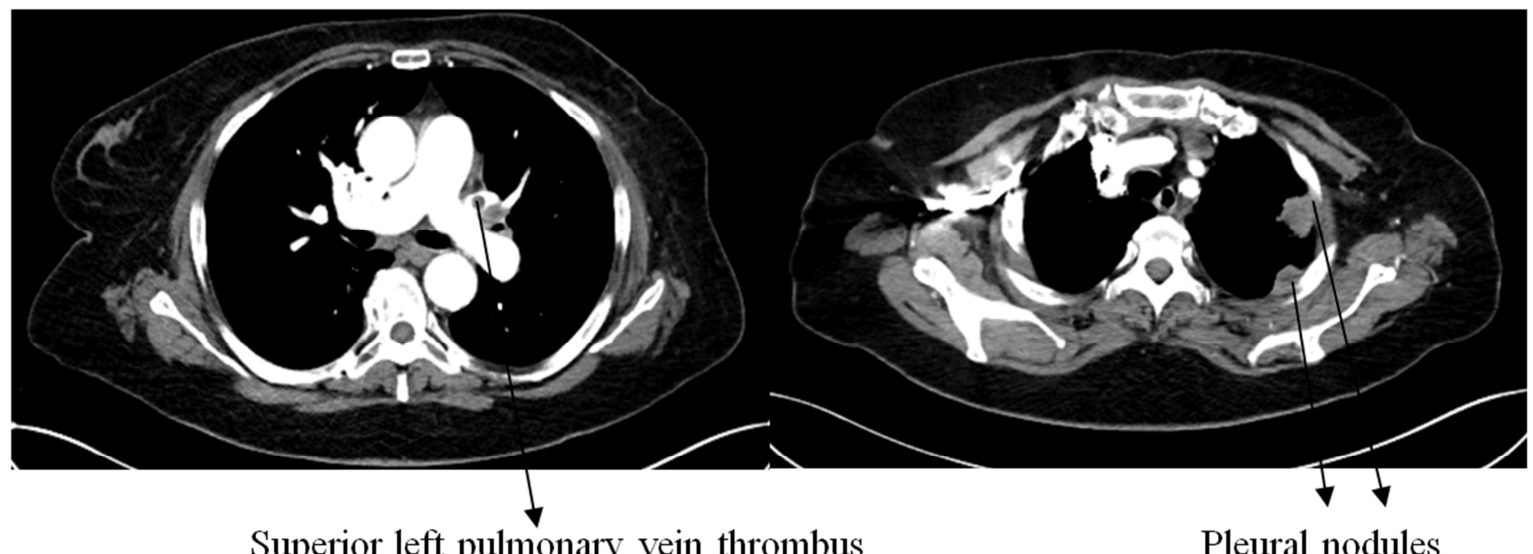

Figure 3. Chest CT with intravenous contrast showing a filling defect within the superior left pulmonary vein consistent with a venous thrombus and multiple pleural nodules.
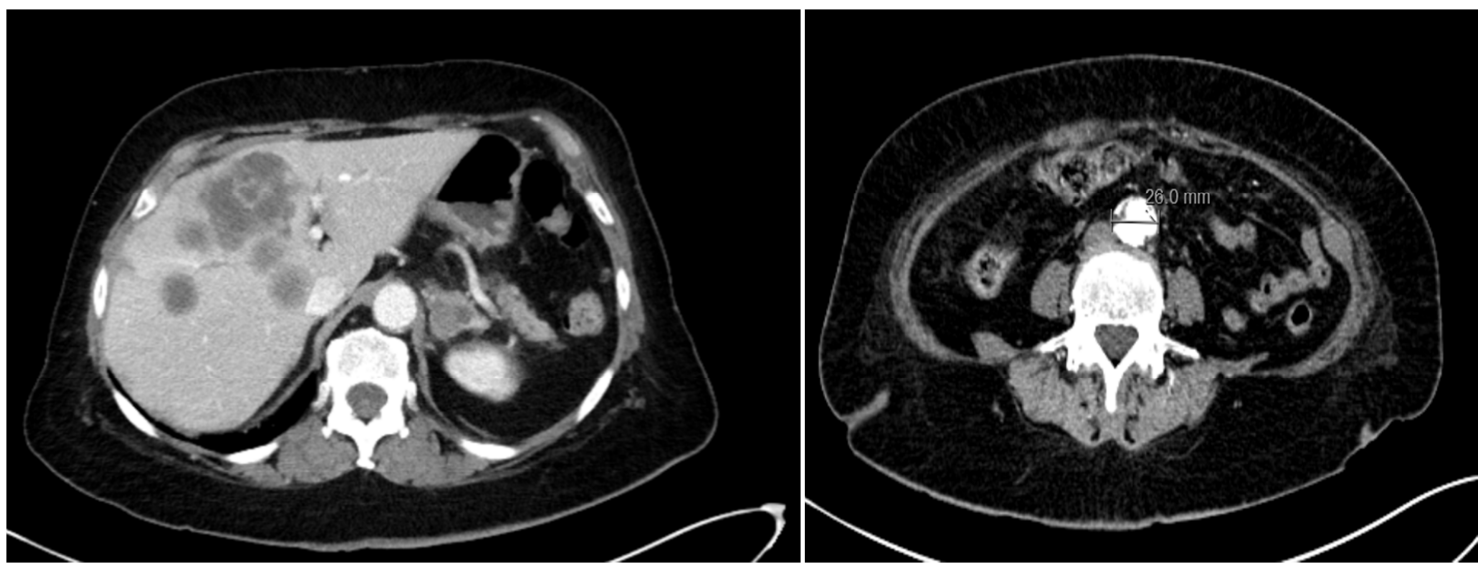

Figure 4. Contrast CT of the abdomen revealing multiple hypoenhancing ill-defined hepatic masses and mild fusiform dilatation of infra-renal aorta. 


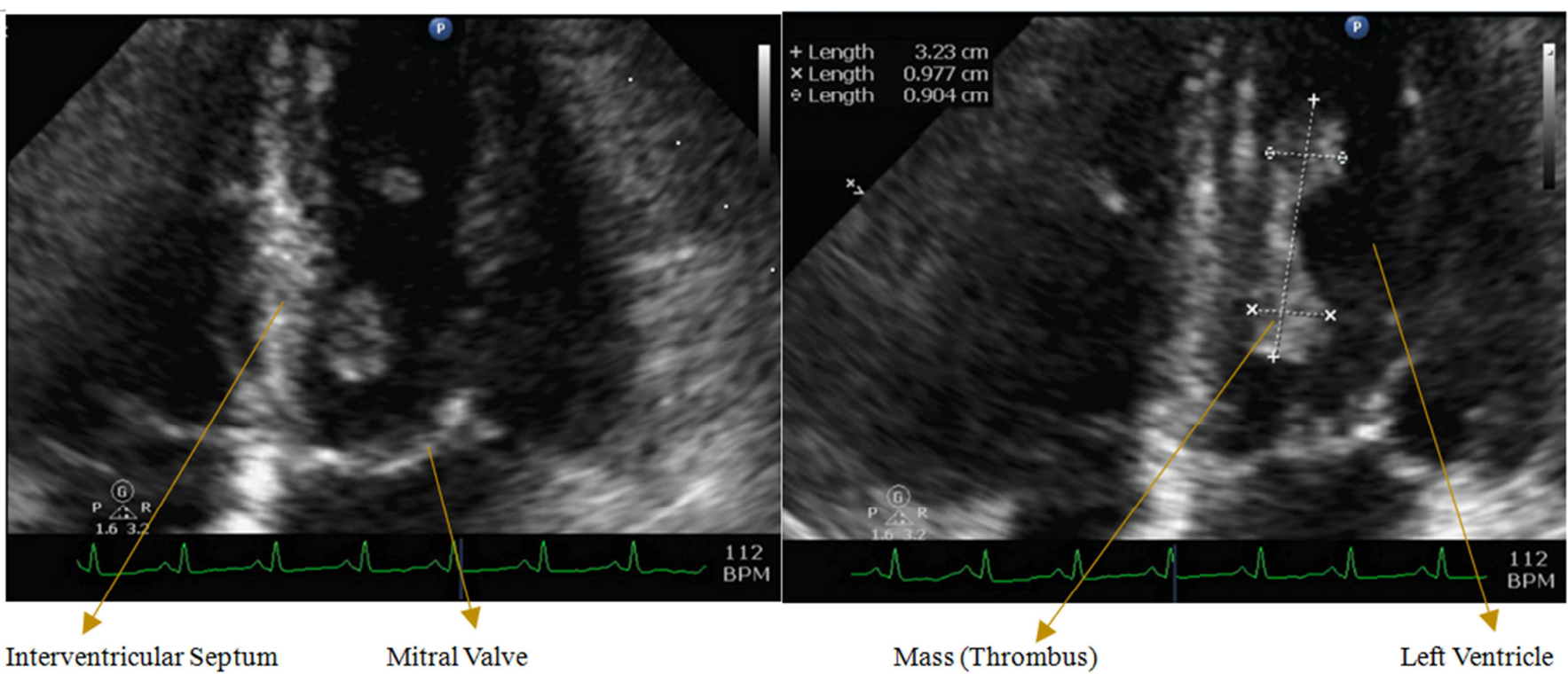

Figure 5. 2D Echocardiogram revealing a large $(3.23 \times 0.98 \mathrm{~cm})$ mass in the left ventricular cavity in close apposition to the interventricular septum, consistent with thrombus.

Post-operatively she developed multi-organ dysfunction including respiratory failure requiring endotracheal intubation and mechanical ventilation, acute kidney injury (with $3+$ hematuria and $2+$ proteinuria), cerebral dysfunction and shock requiring vasopressors. Her platelet count dropped from 298,000 three weeks prior to presentation to $119,000 /$ micro liter on admission. Because the patient was taking enoxaparin and had a 4T's score of 5, there was concern for heparin-induced thrombocytopenia, hence anticoagulation was changed from enoxaparin to argatroban.Her poor prognosis was discussed with her family, who asked for comfort care only and withdrawal of mechanical ventilation. The patient had terminal weaning from mechanical ventilation and she died on the sixth day of admission.

\section{Discussion}

Venous thromboembolism (VTE) is a common complication of malignancy. In most cases, thromboembolic events occur in the setting of a clinically evident malignancy ${ }^{[8,9]}$. Clinically apparent VTE occurs in as many as 10 percent of patients with cancer ${ }^{[10-13]}$. VTE typically presents as deep vein thrombosis (DVT) and/or pulmonary embolism (PE), in adults with cancer; PVT in patients with cancer is rare. PVT is difficult to diagnose clinically because of its non-specific signs and symptoms. A combination of conventional diagnostic modalities such as ventilation-perfusion scanning, pulmonary angiography, bronchoscopy, transthoracic echocardiography (TTE), trans-esophageal echocardiography (TOE), computed tomography (CT) and magnetic resonance imaging (MRI) is often required to make a diagnosis of $\mathrm{PVT}^{[1-7]}$.

The cause of PVT is unclear. Direct extension of tumor into proximal venules may lead to stasis and thrombosis of the larger distal veins. Iatrogenic or immunologic damage to the vein walls may also promote thrombosis after lung surgery. The pathophysiology of PVT is analogous to that of mitral stenosis ${ }^{[14]}$. A significant decrease in the mitral valve area causes an increase in the left atrioventricular gradient and pulmonary venous pressure with compensatory pulmonary arteriolar vasoconstriction. "Leaky capillaries," interstitial edema, and increased uptake by lymphatics through the mechanism of Starling forces occur. Elevated pulmonary artery pressure, right ventricular end-diastolic pressure, and right ventricular dilatation may ultimately result.

Risk factors for recurrent VTE in patients with malignancy despite anticoagulation include poor adherence to medication, inadequate dosing, presence of heparin-induced thrombocytopenia, inherited thrombophilia, interruption of therapy for procedures, drug (e.g. tamoxifen, bevacizumab), prolonged immobilization, heart failure, smoking, inflammatory bowel disease, presence of metastases and creatinine clearance $<30 \mathrm{~mL} / \mathrm{min}^{[15]}$.

Management of patients with recurrent VTE despite anticoagulant therapy is poorly studied. Similar to patients without cancer, patients with symptomatic recurrence are often taking sub-therapeutic doses of their medication. Recurrence in the setting of adequate anticoagulation is more worrisome, indicating drug failure or resistance. Management options include treatment with an alternate agent, escalation of the dose of vitamin $\mathrm{K}$ antagonist or low molecular weight heparin (LMWH), or addition of a vena cava filter [16]. Options of therapy for recurrent VTE in the setting of adequate anticoagulation include using an alternate agent, and increasing the dose or frequency of administration. Recurrences occurring in patients on warfarin that is in the therapeutic range should generally be switched to LMWH; recurrences on a single daily dose of LMWH can be switched to every 12 hour administration at a therapeutic dose or increased to 120 percent of the therapeutic dose ${ }^{[17]}$. 
The development of systemic embolization in our patient who had been on therapeutic anticoagulation with enoxaparin for three weeks prior to presentation; and the greater than $50 \%$ decrease in platelet count and a 4T's pretest clinical score ${ }^{[18]}$ raised suspicion for heparin-induced thrombocytopenia (HIT), hence enoxaparin was discontinued and argatroban was started. The platelet heparin antibody (PF4) was indeterminate, with a value of 0.37 . HIT was excluded as a contributing factor to this patient's presentation by a negative serotonin release assay. The large thrombus found in the left ventricle most likely represents a thrombus in transit from the superior left pulmonary vein and not an in situ intracardiac thrombus, given the normal left ventricular systolic function and absent spontaneous echo contrast in the left ventricle.

\section{Conclusion}

This case describes the occurrence of systemic embolization despite anticoagulation for pulmonary vein thrombosis in a patient with a history of treated cancer, presenting with features suggestive of metastases. The large thrombus found in the left ventricle most likely represents a thrombus in transit from the superior left pulmonary vein enroute systemic embolization with consequent multi-organ dysfunction.

\section{References}

[1] Dye TE, Saab SB, Almond CH, Watson L.Sclerosing mediastinitis with occlusion of pulmonary veins: manifestation and management. $J$ Thorac Cardiovasc Surg 1977, 74:137-141.

[2] Stevens LH, Hormuth DA, Schmidt PE, Atkins S, Fehrenbacher JW. Left atrial myxoma: pulmonary infarction caused by pulmonary venous occlusion. Ann Thorac Surg 1987, 43:215-217.

[3] Mario JG, Leonardo R, Pieter V. Pulmonary vein thrombosis and peripheral embolization. Chest 1996, 109:846-847.

[4] Sarsam MA, Yonan NA, Beton D, McMaster D, Deiraniya AK Early pulmonary vein thrombosis after single lung transplantation. J Heart Lung Transplant 1993, 12:17-19.

[5] McIlroy DR, Sesto AC, Buckland MR. Pulmonary vein thrombosis, lung transplantation and intraoperative transesophageal echocardiography. $J$ Cardiothorac Vasc Anesth 2006, 20(5):712-715
[6] Dore R, Alerci M, D'Andrea F, Di Giulio G, De Agostini A, Volpato G. Intracardiac extension of lung cancer via pulmonary veins: CT diagnosis. J Comput Assist Tomogr 1988, $12: 565-568$

[7] Cavaco R, Kaul S, Chapman T, Casaretti R, Philips B, Rhodes A, Grounds MR. Idiopathic pulmonary fibrosis associated with pulmonary vein thrombosis: a case report. Cases Journal 2009, 2:9156

[8] Goldenberg N, Kahn SR, Solymoss S. Markers of coagulation and angiogenesis in cancer-associated venous thromboembolism. J Clin Oncol 2003; 21:4194-4199.

[9] Maraveyas A, Johnson M. Does clinical method mask significant VTE-related mortality and morbidity in malignant disease? Br J Cancer 2009; 100:1837-1841.

[10] Timp JF, Braekkan SK, Versteeg HH, Cannegieter SC. Epidemiology of cancer-associated venous thrombosis. Blood 2013; 122:1712-1723.

[11] Pabinger I, Thaler J, Ay C. Biomarkers for prediction of venous thromboembolism in cancer. Blood 2013; 122:20112018.

[12] Barsam SJ, Patel R, Arya R. Anticoagulation for prevention and treatment of cancer-related venous thromboembolism. $\mathrm{Br}$ J Haematol 2013; 161:764-777.

[13] Falanga A, Marchetti M, Vignoli A. Coagulation and cancer: biological and clinical aspects. J Thromb Haemost 2013; 11:223-233.

[14] Wyatt PJ, Burke RD, Hanlon RC. Morphologic study of canine lungs after ligation of the pulmonary veins. Am J Pathol 1953; 29: 291-303

[15] Trujillo-Santos J, Nieto JA, Tiberio G, et al. Predicting recurrences or major bleeding in cancer patients with venous thromboembolism. Findings from the RIETE Registry.J Thromb Haemost 2008; 100:435-439.

[16] Lyman GH, Khorana AA, Kuderer NM, et al. Venous thromboembolism prophylaxis and treatment in patients with cancer: American Society of Clinical Oncology clinical practice guideline update. J Clin Oncol 2013; 31:2189-2204.

[17] Carrier M, Le Gal G, Cho R, et al. Dose escalation of low molecular weight heparin to manage recurrent venous thromboembolic events despite systemic anticoagulation in cancer patients. $J$ Thromb Haemost 2009; 7:760-765.

[18] Lo GK, Juhl D, Warkentin TE, et al. Evaluation of pretest clinical score (4 T's) for the diagnosis of heparin-induced thrombocytopenia in two clinical settings. $J$ Thromb Haemost 2006; 4:759-765. 